\title{
Cognitive Effects of Neonatal Hippocampal Lesions in a Rat Model of Schizophrenia
}

\author{
R. Andrew Chambers, M.D., Jonathan Moore, B.S., Joseph P. McEvoy, M.D., and Edward D. Levin, Ph.D
}

Lesioning the ventral hippocampus of neonatal rats has been proposed as an experimental model of schizophrenia. This lesion causes a syndrome of hyperresponsivity to the stimulant effects of amphetamine, impaired grooming and disrupted social interactions, effects that emerge during adolescence, much like schizophrenia. Persisting cognitive effects of neonatal ventral hippocampal lesions were assessed in the current study, because the hippocampus is critically important for a variety of cognitive functions and cognitive impairment and because it is an important feature of schizophrenia. Spatial learning and working memory were assessed in the radial-arm maze, which is sensitive to the adverse effects of hippocampal lesions made in adults. Lesioned rats showed pronounced deficits in radial-arm maze choice accuracy that persisted throughout training. Deficits were seen during the prepubertal period as well as in adulthood. Even though the lesioned rats performed more poorly, they were significantly less sensitive to the amnestic effects of the nicotinic antagonist mecamylamine and the muscarinic antagonist scopolamine. No significant effects of nicotine or amphetamine were seen in either the lesioned or control groups. The long-lasting deficits in spatial learning and working memory resulting from neonatal ventral hippocampal lesions show that, unlike frontal cortical lesions during the same age, the effects of hippocampal lesions are not overcome during development. The resistance to the amnestic effects of nicotinic and muscarinic acetylcholine (ACh) antagonists suggests that the hippocampus is a critical site for the action of these drugs. Neonatal hippocampal lesions may provide a good model of the cognitive impairments of schizophrenia and may be useful to assess novel drug effects to counteract the cognitive deficits in schizophrenia. (C) 1996 American College of Neuropsychopharmacology

[Neuropsychopharmacology 15:587-594, 1996]
KEY WORDS: Hippocampus; Neonatal; Lesion; Learning; Memory; Schizophrenia; Radial-arm Maze

The hippocampus has been shown in a plethora of studies to be critically important for spatial learning and working memory performance (Jarrard 1993). Neonatal lesions of hippocampal connections have been found to cause selective deficits in spatial alternation learning when the rats were tested during the postweaning period (Freeman and Stanton 1991). The de-

From the Department of Psychiatry, Duke University Medical Center, Durham, North Carolina.

Address correspondence to: Dr. Edward D. Levin, Neurobehavioral Research Laboratory, Box \#3412, Department of Psychiatry, Duke University Medical Center, Durham, NC 27710.

Received January 19, 1996; revised May 8, 1996; accepted May 15 1996. veloping organism shows considerable plasticity and ability to recover from neural lesions. For example, recovery from certain types of neonatal frontal cortical lesions can be fairly complete in terms of working memory performance (Kolb and Sutherland 1992). However, recovery from subcortical damage may differ. The current study examined the persisting cognitive effects of neonatal ventral hippocampal lesions and the involvement of nicotinic and muscarinic acetylcholine (ACh) systems in these effects.

Neonatal ventral hippocampal neurotoxic lesions have been developed by Lipska and Weinberger as a rat model of schizophrenia (Lipska et al. 1993; Lipska and Weinberger 1993). This model mimics perinatal abnormality of hippocampal structures thought to be present in schizophrenic brains. More important, the model 
shows the emergence of symptoms in rats after adolescence (Lipska et al. 1983). Lesioned rats become hyperresponsive to stress and amphetamine and show attenuation to these effects with haloperidol (Lipska et al. 1993; Lipska and Weinberger 1993). They also show postpubertal abnormalities in habituation to acoustic startle (Lipska et al. 1995b). Further investigation has revealed alteration of midbrain dopamine (DA) systems during stress or pharmacological challenge and differential effects of clozapine versus haloperidol (Lipska et al. 1995a, Lipska and Weinberger 1994).

The current study was designed to assess the lasting impact of neonatal ventral hippocampal lesions on cognitive performance, specifically spatial learning and working memory. Such investigation is pertinent to the modeling of attentional and memory disturbances observed in schizophrenia (Cornblatt and Keilp 1994; Duffy and Ocarroll 1994). Nicotinic and muscarinic ACh agonist and antagonist effects on memory in these rats were examined because of important $\mathrm{ACh}$ interactions with DA systems underlying memory (Levin and Rose 1992). In addition, we may better understand the high rates of nicotine use in schizophrenia that may reflect self-medication for such cognitive disturbance (Lohr and Flynn 1992).

The radial-arm maze (RAM) was used to assess spatial learning and working memory in the current experiment. It has been found to provide a sensitive assessment of learning and working memory in rats exposed to a variety of psychoactive drugs (Levin 1988) and hippocampal lesions in adult rats (Olton et al 1978)). Both nicotinic and muscarinic $\mathrm{ACh}$ agonists have been shown to enhance choice accuracy performance on the RAM while their antagonists impair performance (Levin 1988, 1992). Interactions between drugs active as agonists and antagonists at $\mathrm{ACh}$ and DA receptors of various subtypes have also been observed (Levin and Rose 1992). Therefore, we tested the effects of ACh drugs on neonatal ventral hippocampal lesioned rats. Pharmacological manipulation of ACh systems could carry implications relevant to the hypothesis of ACh hyperactivity in schizophrenia (Tandon and Greden 1989).

\section{MATERIALS AND METHODS}

\section{Subjects}

Five cohorts of Sprague-Dawley rats (Zivic-Miller Labs, Allison Park, PA) were tested in this study. Three cohorts were born in the lab to primiparous mother rats obtained at 16- to 18-day's gestation. Two cohorts arrived as newborn same-sex litters. Upon arrival, the pregnant rats were separated into individual cages and fed ad libitum under a 12-hour reverse day-light cycle (lights on at 6:00 P.M.). The rats delivered their pups and were left undisturbed until postnatal day 7 (PND 7) when the entire litter was removed for surgery. Those rats that arrived in litters were likewise kept with their mothers under these conditions until PND 7 when they also were removed for surgery.

\section{Surgery}

Those rats weighing 15 to $20 \mathrm{~g}$ were randomized to receive either sham or neurotoxin administration. The animals were anesthetized by induction of hypothermia by being kept on ice for 15 to 20 minutes. They were then secured with tape to a stereotaxic platform. Surgery consisted of an incision made into the dorsal aspect of the subject's skull, followed by placement of a Hamilton needle into the ventral hippocampal formation bilaterally at coordinates AP $-3.0 \mathrm{~mm}, \mathrm{ML} \pm 3.5 \mathrm{~mm}$, and VD $\pm 5.0 \mathrm{~mm}$ relative to bregma (Lipska et al 1993). The same needle was used on both sides of the brain to deliver $3.0 \mu \mathrm{g}$ of ibotenic acid (Sigma, St. Louis, MO) in a volume of $0.3 \mu \mathrm{l}$ of artificial cerebrospinal fluid (CSF) vehicle. The excitotoxin was delivered over a period $s$ of $129 \mathrm{~s}$ as set on an infusion pump. Sham lesioned rats received only artificial CSF. The needle was left in place for 3 minutes after cessation of infusion to prevent flow of neurotoxin/vehicle through the needle track. The surgical wound was closed using Nexaband veterinary cement and the pups were warmed with a heating pad before reunion with their mothers. The litters were again maintained undisturbed under conditions as before the surgery until PND 23 when the pups were weaned and placed randomly with regard to lesion status into samesex groups of two to three. Rats were kept in such quarters for the duration of their behavioral testing.

\section{Behavioral Testing}

The radial-arm maze (RAM), was a black painted wooden structure. It had a central arena $50 \mathrm{~cm}$ in diameter and eight $10-\times 60-\mathrm{cm}$ runways extending radially. Placed 2 $\mathrm{cm}$ from the end of each runway and hidden from view by a small wooden block was the food reward. The maze stood $30 \mathrm{~cm}$ above the floor and was surrounded by a variety of visual landmarks in a quiet room. Behavioral testing assessed working memory by use of the win-shift paradigm as described by Olton and Samuelson (Olton and Samuelson 1976). In this paradigm, rats are placed on the maze with all arms loaded with $1 / 3$ to $1 / 2$ of a Kellogg's Froot Loop ${ }^{\circledR}$. After $10 \mathrm{~s}$ of confinement in an opaque central arena, the rat was allowed to seek food reward until either all 8 arms had been entered or $300 \mathrm{~s}$ had passed. The criterion for an arm entry was that a rat had placed all four paws beyond the border of the central arena and the radially extending runway. Measures recorded for a session include Entries to Repeat (ETR) and Latency (LAT). ETR, the measure of choice accuracy, was defined as the number of different 
arms traversed before entry into an arm is repeated. LAT, a measure of locomotor activity, was defined as the time the rat took to complete the maze (enter all eight arms) divided by the number of entries made. There was a maximum 300-s session length.

The rats were trained on the RAM four to five times a week for a total of 18 sessions. The rats began testing at prepubertal (PND 25), pubertal (PND 40), and postpubertal (PND 80) ages. One cohort (lesioned $N=7$, sham $N=8$ ) began training at PND 25. Three cohorts (Lesioned $N=10$, sham $N=27$ ) began training at PND 40. Another cohort (Lesioned $N=8$ sham $N=8$ ) began training at PND 80. In the second phase, three cohorts previously trained on the RAM were again assessed on the RAM as adults (after PND 70) for dose-response assessment with nicotine, mecamylamine, scopolamine, or amphetamine. Two cohorts (lesion $N=14$, sham $N=$ 18 ), both of which were initially trained on the RAM beginning on PND 40, received nicotine, mecamylamine, and then scopolamine in consecutive repeated-measures counterbalanced studies. Doses for each drug were as follows: nicotine, $0,0.1,0.2$, and $0.4 \mathrm{mg} / \mathrm{kg}$; mecamylamine, $0,2.5,5.0$, and $10.0 \mathrm{mg} / \mathrm{kg}$; and scopolamine, $0,0.04,0.08$, and $0.16 \mathrm{mg} / \mathrm{kg}$. A third cohort (lesioned $N=8$, sham $N=8$ ) initially trained on the RAM beginning on PND 25, were given $d$-amphetamine sulfate subcutaneously $(0,0.5,1.0$, and $2.0 \mathrm{mg} / \mathrm{kg})$ in a repeated-measures counterbalanced study. Doses of all drugs were given as subcutaneous injections $(1 \mathrm{mg} / \mathrm{ml})$ 20 minutes before RAM testing. Rats were allowed a drug washout period of at least 2 days between doses. The testers were blind to treatment condition of the rats.

\section{Data Analysis}

Analysis of variance (ANOVA) for within and between measures was used for statistical analysis. The betweensubjects factors were sex, age, and lesion treatment, and the within-subjects factors were week of testing and drug dose. For the acquisition phase, unilateral lesions were compared to bilateral lesions and to sham lesions. For the drug challenge phases, only the bilateral lesions and sham lesions were compared. Dependent measures were entries to repeat and seconds per entry. A $p$ value of .05 was used as a cutoff for statistical significance. Two-tailed tests were used throughout. The data are presented as mean \pm standard error of the mean (SEM).

\section{RESULTS}

\section{Lesion Verification}

The brains of all animals in the study were sectioned and Nissl stained (Figure 1). As expected, the brains of rats receiving sham surgeries (45 rats) showed no evidence of abnormality. The brains of rats receiving exci- totoxin (47 rats) showed damage to the hippocampus bilaterally in 27 cases, unilaterally in 13 cases, and negligible damage to either side in 7 cases. The effect of unilaterally versus bilateral lesion effects during acquisition is shown in Figure 2 to convey the finding that rats with this lesion had attenuated choice accuracy deficits. Only those with bilateral hippocampal damage were used for data analysis of the age and drug effects. Recognizable damage was most often confined to the ventral hippocampus, which revealed neuronal loss in CA1 and CA3 cellular layers and atrophy of surrounding hippocampal tissue. The lateral ventricle was commonly enlarged. Damage to extrahippocampal tissue included evidence of mild gliosis in lateral thalamic structures adjacent to the hippocampus in three cases and more anteriorly cavitation in the area of the caudate-putamen in one case. In six other cases there was atrophy of structures of the amygdala adjacent to an enlarged lateral ventricle. Review of the data revealed that rats receiving extrahippocampal damage did not perform differently from those showing damage confined strictly to the ventral hippocampus.

\section{Radial-Arm Maze Acquisition}

Both the lesioned and unlesioned rats started at similar levels of choice accuracy at the beginning of training (Figure 2). Subsequently, the lesioned rats had a pronounced and persistent deficit in choice accuracy. For comparison, performance of the rats with unilateral hippocampal lesions is shown. They had clearly impaired performance, but the deficit was not as severe as that in the rats with bilateral lesions. Pairwise comparisons using the Tukey procedure showed that both unilateral and bilateral lesioned rats were significantly worse than sham lesioned controls $(p<.0001)$ but that the unilateral lesioned rats were significantly less impaired than the bilateral lesioned rats $(p<.05)$. Statistical analysis of the acquisition and drug challenge data compared the effects of bilateral lesions versus sham lesioned controls, as there were not enough unilateral lesioned rats in each age group to include in the analysis. There were significant main effects of lesion $[F(1,56)=$ 78.84, $p<.0001]$, age of testing $[F(2,56)=8.46, p<$ $.001]$, and trial block $[F(5,280)=45.98, p<.0001]$. Post hoc Tukey's comparisons showed that the PND 40 age group performed significantly better $(p<.05)$ than the younger or older age groups. The lesion effect on choice accuracy was clearly seen in all three age groups (Figure 3 ). The average entries to repeat scores (mean \pm SEM) during acquisition training were: PND 25, sham = $6.01 \pm 0.15$, lesioned $=4.19 \pm 0.23, p<.001 ;$ PND 40, sham $=6.64 \pm 0.10$, and lesioned $=5.13 \pm 0.27, p<$ $.001 ;$ PND 80, sham $=5.90 \pm 0.29$ and lesioned $=4.60 \pm$ $0.12, p<.005$. There was a significant interaction of lesion and session block $[F(5,280)=5.21, p<.001]$. Tests 

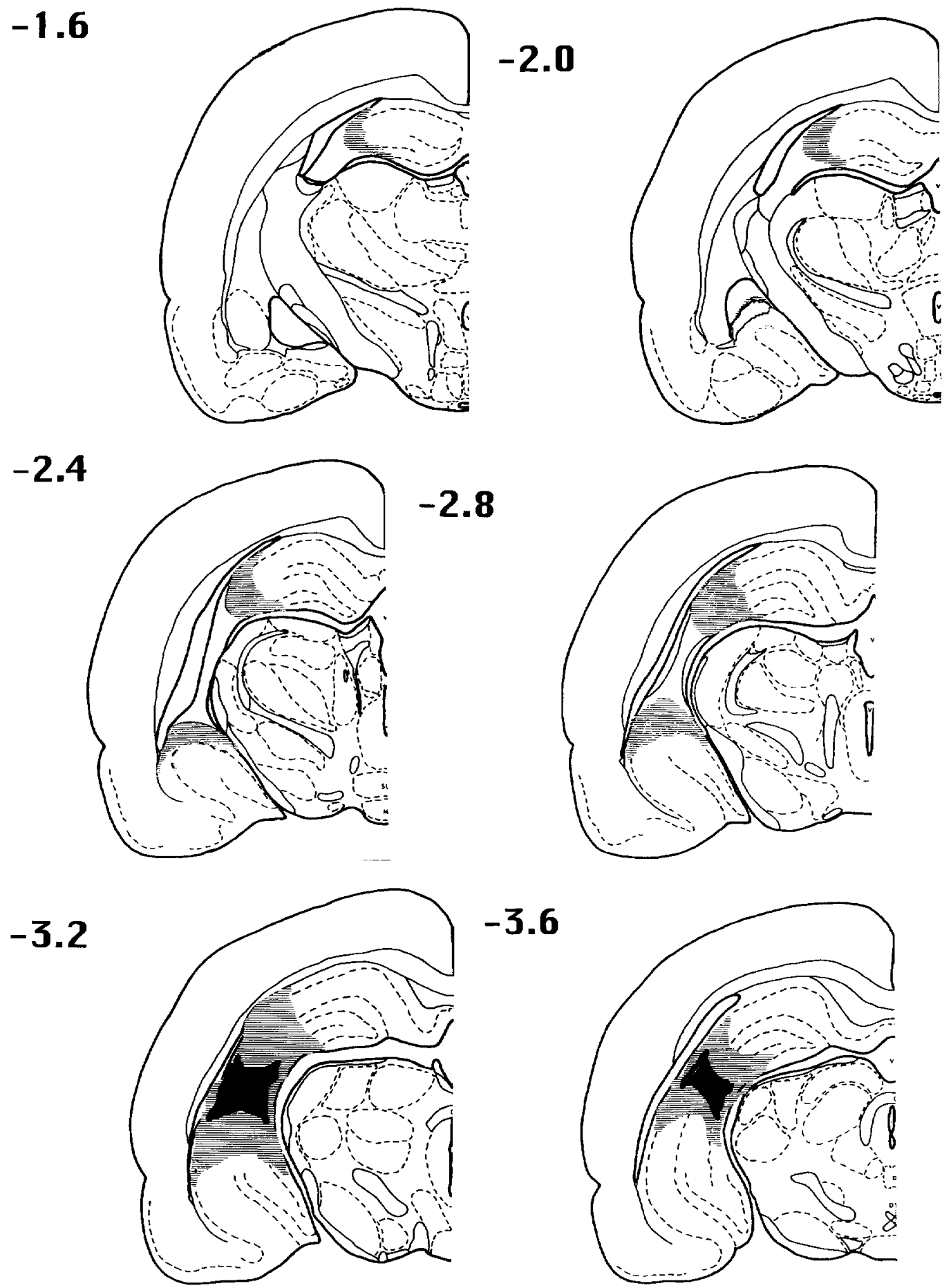

Figure 1. Areas of least and greatest hippocampal damage as determined by Nissl stain in rats receiving ibotenic acid. Numbers refer to measurements (mm) relative to bregma (Pelligrino et al. 1979) Gray, neuronal loss and gliosis in the hippocampus of the rat with the most widespread damage. Black, extent of the lesion in the rat with the minimal damage considered significant. Histology showed damage to range between these extremes in 27 rats receiving significant bilateral damage and 13 receiving significant unilateral damage. Some rats with large hippocampal lesions had unilateral extrahippocampal damage: one anterior caudate-putamen, three lateral thalamus, and six amygdala. 


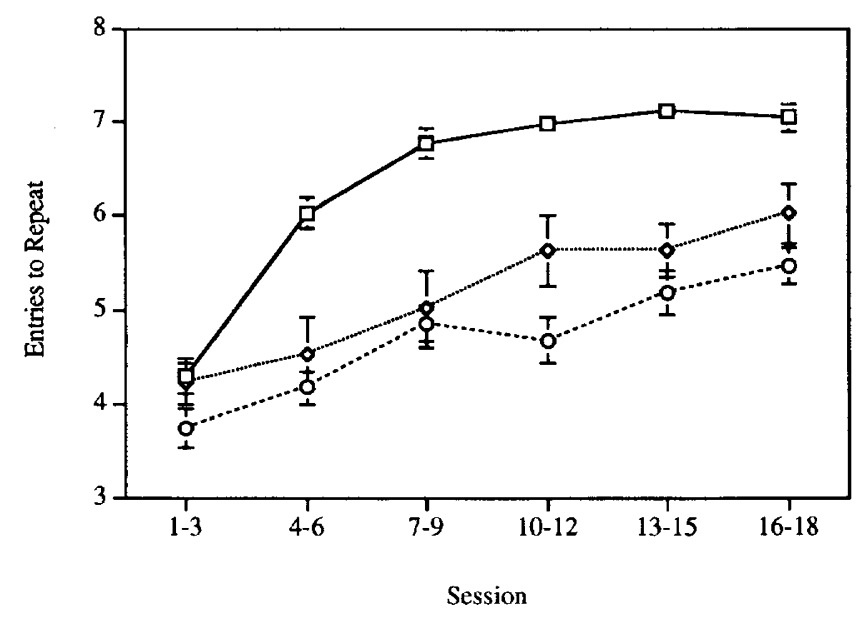

Figure 2. Radial-Arm maze Acquisition, Unilateral and Bilateral Lesion Effects, Entries to Repeat (mean \pm SEM). Squares, Sham; diamonds, unilateral lesions; circles, bilateral lesions.

of the simple main effects showed that there were highly significant $(p<.001)$ deficits in performance by the lesioned rats in all session blocks except the first where there was only a nearly significant deficit $(p<$ .08). Latency was not significantly affected by neonatal hippocampal lesions. The sham-lesioned controls averaged (mean \pm SEM) during acquisition $24.8 \pm 2.1 \mathrm{~s} / \mathrm{en}-$ try, whereas the lesioned rats averaged $28.0 \pm 4.3 \mathrm{~s} /$ entry. No significant effects of sex or sex-related interactions were seen with the choice accuracy or latency measures during acquisition.

\section{Acute Drug Challenges}

Nicotine. No significant effects of nicotine on choice accuracy were seen in either the control or lesioned group. There was a significant lesion-induced deficit $[F(1,26)=20.72, p<.0001]$ with the sham lesioned group averaging $7.21 \pm 0.20$ entries to repeat and the hippocampal lesioned group averaging $5.43 \pm 0.33$ entries to repeat (mean $\pm S E M$ ). Controls were performing at nearly perfect levels even in the saline condition $(7.42 \pm 0.30)$. Little further improvement was possible, and none was seen with nicotine. Control performance at the $0.4-\mathrm{mg} / \mathrm{kg}$ nicotine dose averaged $7.10 \pm 0.34 \mathrm{en}-$ tries to repeat. The lesioned rats had substantially lower scores after saline injections $(5.64 \pm 0.61)$ than controls. No improvement was seen with nicotine in the lesioned rats, even though there was considerable room for improvement. The lesioned rats averaged $5.46 \pm 0.54$ entries to repeat at the $0.4-\mathrm{mg} / \mathrm{kg}$ dose of nicotine. No nicotine or lesion-related effects on response latency were seen during this phase (Table 1).

Mecamylamine. There was a significant main effect of the nicotinic antagonist mecamylamine $[F(3,69)=$ $7.53, p<.001]$. Three rats did not perform under the in- fluence of mecamylamine and were excluded from analysis. There was a significant macamylamine-induced deficit in the sham lesioned subjects $(p<.001)$, but not in the rats with lesions (Figure 4). There was a significant overall lesion-induced choice accuracy deficit $[F(1,23)=5.16 p<.05]$, with the sham lesioned group averaging $6.06 \pm 0.17$ entries to repeat and the hippocampal lesioned group averaging $4.97 \pm 0.38$ entries to repeat (mean \pm SEM). There also was a significant main effect of mecamylamine increasing response latency $[F(3,69)=27.88, p<.0001]$. No differential effects were seen with latency in the sham operated and lesioned groups (Table 1).

Scopolamine. There was a significant main effect of the muscarinic antagonist scopolamine $[F(3,69)=3.48$, $p<.025]$. Three rats did not perform under the influence of scopolamine and were excluded from analysis. There was a significant deficit in the control subjects $(p<$ .001 ), but not in those with lesions (Figure 5). Scopolamine also caused a significant increase in response latency $[F(3,69)=7.19, p<.001]$. Both the sham and lesioned groups had similar scopolamine-induced increases in latency (Table 1).

Amphetamine. No significant effects of amphetamine on choice accuracy were seen in either the control or lesioned group. There was a nearly significant effect of lesion during this phase $[F(1,11)=4.04, p<.07]$. Controls averaged $6.38 \pm 0.48$ entries to repeat and the lesioned rats averaged $4.82 \pm 0.48$ entries to repeat (mean \pm SEM). No amphetamine or lesion effects on response latency were seen (Table 1). No significant effects of sex or sex-related interactions were seen with the choice accuracy or latency measures during the drug challenges.

\section{DISCUSSION}

Ventral hippocampal lesions made 1 week after birth caused a pronounced deficit in spatial learning and memory that persisted into adulthood. The deficit was clearly evident in both males and females and in both pre-and postpubertal rats. The lesioned rats showed attenuated response to the amnestic effects of mecamylamine and scopolamine, nicotinic and muscarinic $\mathrm{ACh}$ antagonists. The deficit was not exacerbated by amphetamine. These data provide important information concerning the nature of neonatal ventral hippocampal lesions on cognitive function and the potential utility of this lesion as a model of schizophrenia.

The current study extends the results from Lipska and Weinberger (Lipska et al. 1993, 1995a, 1995b; Lipska and Weinberger 1993, 1994) in several ways. Using their paradigm, we found that neonatal ventral hippocampal lesions cause lasting deficits in learning and working 


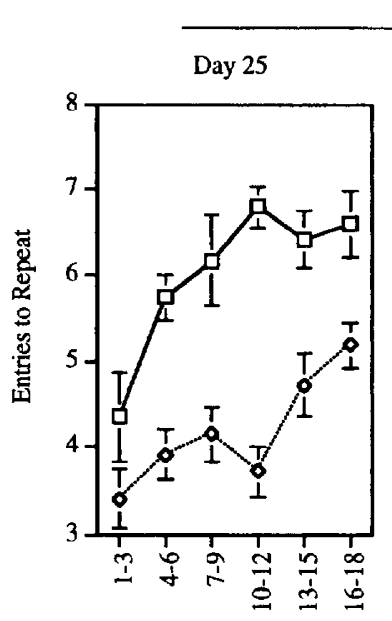

Session
Training Start Day

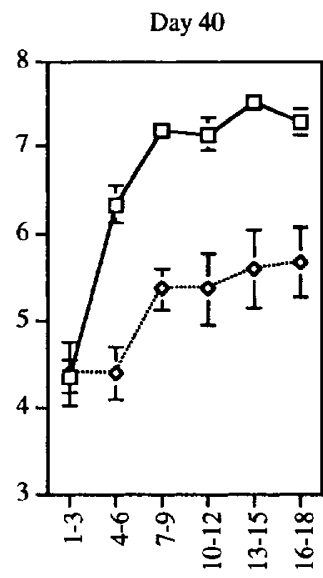

Session

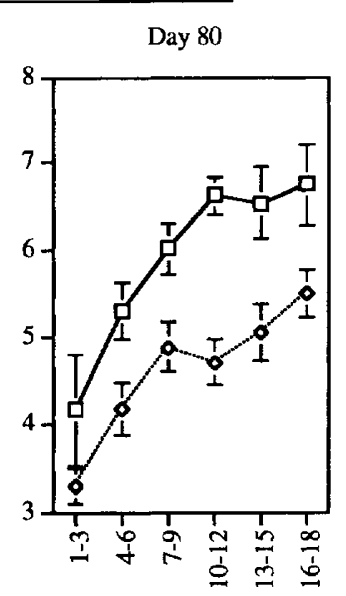

Session
Figure 3. Radial-Arm Maze Acquisition, Age Effects, Entries to Repeat (mean \pm SEM). Squares, sham; diamonds, bilateral lesions. memory. This effect is quite large and is apparent in both male and female rats even with prolonged training. In contrast to the effects on locomotor activity, it does not emerge during the juvenile period but is clearly evident to its full extent during the prejuvenile period. The deficit was seen no matter whether the rats begin training in a prejuvenile (PND 25), juvenile (PND 40 ), or adult age (PND 80). This lack of delayed emergence does not mirror the typical pubertal emergence of schizophrenia This may be taken as a stroke against this model of schizophrenia. But it also may be an advantage of the model. One prospect for a developmental model of schizophrenia is the identification of markers that would predict later emergence of schizophrenic symptoms. The prepubertal learning and memory deficit in this model may be such a predictive marker. As such, it may suggest useful ways to identify people prone to schizophrenia before they develop it, and it may provide a forum in which to test treatments to prevent the development of schizophrenia. Cornblatt and Keilp (1994) have found that people prone to develop schizophrenia have impaired attentional processes. The mildly stressful circumstances associated with the daily feeding schedule may have contributed to the early appearance of the lesion-induced deficits in this study.

The purpose of the current study was to assess the cognitive effects of ventral hippocampal lesions. Our primary measure of memory was choice accuracy (entries to repeat, ETR). As a control measure latency measures are taken to determine whether impairments in choice accuracy are due to the subject having to remember over longer periods rather than impaired memory. This latency measure is very much unlike usual measures of locomotor activity such as used by Lipska et al. (Lipska et al. 1993; Lipska and Weinberger 1993). In the current study the working memory deficit was not potentiated by amphetamine, which reliably increases activity with classical measures. Therefore it is not surprising that no lesion-induced reduction in response latency was seen in the current study, either in the drug-free or drug-challenged state. The current latency results are therefore not directly comparable to the Lipska data because the rats were tested only for brief periods repeatedly in a maze rather than for longer periods in an

Table 1. Response Latency for Radial-Arm Maze Performance after the Drug Challenges (mean \pm SEM)

\begin{tabular}{|c|c|c|c|c|}
\hline Mecamylamine (mg/kg) & 0 & 2.5 & 5 & 10 \\
\hline Sham & $16.4 \pm 1.1$ & $27.9 \pm 4.2$ & $42.4 \pm 3.6$ & $65.0 \pm 6.6$ \\
\hline Lesioned & $12.0 \pm 0.8$ & $19.3 \pm 2.3$ & $36.2 \pm 3.2$ & $54.1 \pm 7.3$ \\
\hline Nicotine (mg/kg) & 0 & 0.1 & 0.2 & 0.4 \\
\hline Sham & $15.0 \pm 1.9$ & $15.8 \pm 2.0$ & $13.8 \pm 0.9$ & $17.5 \pm 3.6$ \\
\hline Lesioned & $12.2 \pm 0.8$ & $10.8 \pm 0.5$ & $11.7 \pm 0.9$ & $10.9 \pm 0.9$ \\
\hline Scopolamine (mg/kg) & 0 & 0.04 & 0.08 & 0.16 \\
\hline Sham & $16.4 \pm 1.2$ & $28.1 \pm 3.6$ & $36.8 \pm 7.4$ & $41.1 \pm 4.8$ \\
\hline Lesioned & $16.0 \pm 2.6$ & $21.2 \pm 2.6$ & $36.4 \pm 6.5$ & $41.0 \pm 6.7$ \\
\hline Amphetamine (mg/kg) & 0 & 0.5 & 1.0 & 2.0 \\
\hline Sham & $12.8 \pm 1.1$ & $11.6 \pm 0.9$ & $12.0 \pm 0.6$ & $12.2 \pm 0.9$ \\
\hline Lesioned & $11.1 \pm 1.0$ & $11.0 \pm 1.0$ & $9.1 \pm 1.4$ & $10.1 \pm 1.0$ \\
\hline
\end{tabular}




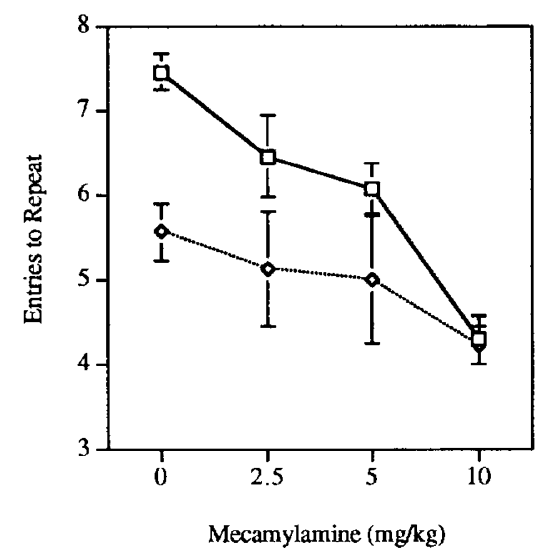

Figure 4. Radial-Arm Maze Acquisition, Mecamylamine Challenge, Accuracy, Entries to Repeat (mean \pm SEM). Squares, sham; diamonds, lesions.

activity arena. Also important, all rats in the current study were under restricted feeding and were tested in the maze while searching for food.

Interestingly, the lesioned rats showed protection against the amnestic effects of both the nicotinic and muscarinic antagonists mecamylamine and scopolamine. This was not due to a floor effect because random-chance choice accuracy using the ETR measure is 3.25. Even with the highest doses of these drugs, performance was well above this floor. This resistance to the amnestic effects of anticholinergic drugs was seen despite the fact that the lesioned rats had a significant deficit in choice accuracy apparent during the acquisition and the drug challenge phases. This attenuated response may have been due to lesions in the critical target for these drugs. In another recent study, we found that administering either of these antagonists directly into the ventral hippocampus caused radial-arm maze

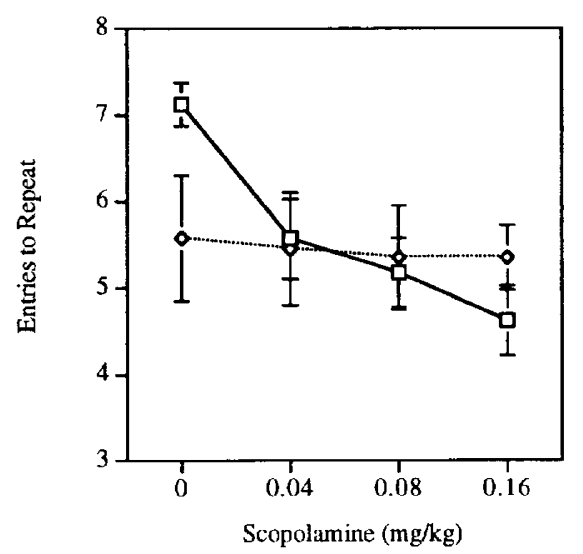

Figure 5. Radial-Arm Maze Acquisition, Scopolamine Challenge, Accuracy Entries to Repeat (mean \pm SEM). Squares, sham, diamonds, lesions. working memory deficits (Kim and Levin 1996). Lesioning the ventral hippocampus in the current study may have eliminated a critical site of action of these drugs. The nicotinic ACh agonist nicotine did not attenuate the lesion effect, which may result from the same loss of a critical target structure. A similar underresponsiveness to the amnestic effects of mecamylamine was seen in a previous study in adult rats of the cognitive effects of medial basalocortical knife cut lesions (McGurk et al. 1991). The lesioned rats tested in the radial-arm maze showed a lack of response to mecamylamine $(10 \mathrm{mg} /$ $\mathrm{kg}$ ) that had previously caused significant memory deficits in these and other rats. In contrast to the effects of ventral hippocampal lesions, medial basalcortical knifecut lesions heightened the amnestic effects of scopolamine.

No differential response to nicotine or amphetamine was seen in the current study. However, this result must be viewed with caution because there were no significant effects of these drugs in the control sham operated rats. In previous studies, we have found this dose range of nicotine to be effective in improving choice accuracy in the radial-arm maze (Levin and Torry 1995). The fact that the control rats administered nicotine were already performing at nearly perfect levels presented a ceiling effect that would have prevented the detection of nicotine-induced improvement. We have seen this effect impair expression of acute nicotine effects previously (Levin and Torry 1996). Various researchers have found amphetamine either to have no effect or to impair memory performance in the radial-arm maze (for review, see Levin 1988). No effect of amphetamine was seen in the controls in the current study, and amphetamine was not seen to exacerbate the deficit shown by the rats with neonatal hippocampal lesions.

The current study provides a characterization of the persisting cognitive effects of neonatal ventral hippocampal lesions. Neonatal ventral frontal cortical lesions in rats 1 week after birth do not cause lasting deficits in radial-arm maze working memory performance, whereas medial frontal cortical lesions do (Kolb 1995; Kolb and Sutherland 1992; Kolb and Whishaw 1985). The current results with ventral hippocampal lesions are more similar to the medial frontal cortical lesions. Comparing the hippocampal lesions of the current study to lesions of other areas of the cortex and subcortical areas such as the striatum would help to complete the picture of the role each of these areas plays in the development of cognitive function.

These results demonstrate the differences in the effects of neonatal ventral hippocampal lesions of cognitive function versus locomotor activity and other behavioral effects seen after this lesion. The learning and memory deficits were seen before sexual maturity and this effect was not potentiated by amphetamine. The early emergence of the cognitive effects of this lesion 
may suggest an early marker for later emergence of other pathological symptoms. The current data also provide important information concerning the neural substrates for learning and memory. The finding that the lesioned rats were resistant to the amnestic effects of the nicotinic and muscarinic ACh antagonists, mecamylamine, and scopolamine added evidence for the hypothesis that the ventral hippocampus is a critical target for these anticholinergic drugs. Further studies should evaluate the relationship between cognitive and noncognitive symptoms as well as further assess the neural foundations of these deficits.

\section{ACKNOWLEDGMENTS}

This project was supported by a grant from the National Alliance for Research on Schizophrenia and Depression.

\section{REFERENCES}

Cornblatt BA, Keilp JG (1994): Impaired attention, genetics, and the pathophysiology of schizophrenia. Schiz Bull 20:31-46

Duffy L, Ocarroll R (1994): Memory impairment in schizophrenia-A comparison with that observed in the alcoholic Korsakoff syndrome. Psychol Med 24:155-165

Freeman JH, Stanton ME (1991): Fimbria-fornix transections disrupt the ontogeny of delayed alternation but not position discrimination in the rat. Behav Neurosci 105: 386-395

Jarrard LE (1993): On the role of the hippocampus in learning and memory in the rat. Behav Neural Biol 60:9-26

Kim J, Levin E (1996): Nicotinic, muscarinic and dopaminergic actions in the ventral hippocampus and the nucleus accumbens: Effects on spatial working memory in rats. Brain Res 725:231-240

Kolb B (1995): Brain Plasticity and Behavior. Mahwah,NJ Erlbaum

Kolb B, Sutherland RJ (1992): Noradreneline depletion blocks behavioral sparing and alters cortical morphogenesis after frontal cortex damage in rats. J Neurosci 12:2321-2330

Kolb B, Whishaw IQ (1985): Earlier is not always better: Behavioral dysfunction and abnormal cerebral morphogenesis following neonatal cortical lesions in the rat. Behav Brain Res 17:25-43

Levin ED (1988): Psychopharmacological effects in the radialarm maze. Neurosci Biobehav Rev 12:169-175
Levin ED (1992): Nicotinic systems and cognitive function. Psychophamacology 108:417-431

Levin ED, Rose JE (1992): The importance of $D_{1}$ and $D_{2}$ interactions with nicotinic and muscarinic systems for working memory function. In Levin ED, Decker MW, Butcher LL (eds), Neurotransmitter Interactions and Cognitive Function. Boston, Birkhäuser, pp 144-158

Levin ED, Torry D (1995): Nicotine effects on memory performance. In Clarke PBS, Quik M, Thurau K, Adlkofer F (eds), International Symposium on Nicotine: The Effects of Nicotine on Biological Systems II. Boston, Birkhäuser, pp 329-336

Levin ED, Torry D (1996): Acute and chronic nicotine effects on working memory in aged rats. Psychopharmacology 123:88-97

Lipska BK, Weinberger DR (1983): Delayed effects of neonatal hippocampal damage on haloperidol-induced catalepsy and apomorphine-induced stereotypic behaviors in the rat. Dev Brain Res 75:213-222

Lipska BK, Weinberger DR (1994): Subchronic treatment with haloperidol and clozapine in rats with neonatal excitotoxic hippocampal damage. Neuropsychopharmacology 10:199-205

Lipska BK, Jaskiw GE, Weinberger DR (1993): Postpubertal emergence of hyperresponsiveness to stress and to amphetamine after neonatal excitotoxic hippocampal damage: A potential animal model of schizophrenia. Neuropsychopharmacology 9:67-75

Lipska BK, Chrapsusta SJ, Egan MF, Weinberger DR (1995a): Neonatal excitotoxic ventral hippocampal damage alters dopamine response to mild repeated stress and to chronic haloperidol. Synapse 20:125-130

Lipska BK, Swerdlow NR, Geyer MA, Jaskiw GE, Braff DL, Weinberger DR (1995b): Neonatal excitotoxic hippocampal damage in rats causes post-pubertal changes in prepulse inhibition of startle and its disruption by apomorphine. Psychopharmacology in 122:35-43

Lohr JB, Flynn K (1992): Smoking and schizophrenia. Schiz Res 8:93-102

McGurk SR, Levin ED, Butcher LL (1991): Impairment of radial-arm maze performance in rats following lesions involving the cholinergic medial pathway: Reversal by arecoline and differential effects of muscarinic and nicotinic antagonists. Neuroscience 44:137-147

Olton DS, Samuelson RJ (1976): Remembrance of places passed: Spatial memory in rats. J Exp Psychol 2:97-116

Olton DS, Walker JA, Gage FH (1978): Hippocampal connections and spatial discrimination. Brain Res 139:295-308

Pelligrino LJ, Pelligrino AS, Cushman AJ (1979): A Stereotaxic Atlas of the Rat Brain. New York, Plenum

Tandon R, Greden JF (1989): Cholinergic hyperactivity and negative schizophrenic systems. Arch Gen Psychiatr 46: $745-753$ 